\title{
Improving Degradation Efficiency of \\ Organic Pollutants through a Self-Powered Alternating Current Electrocoagulation \\ System
}

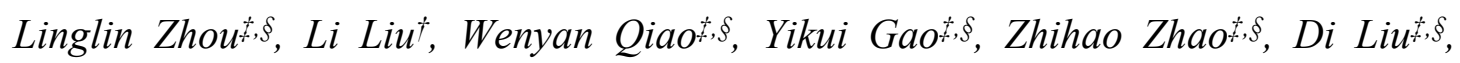
Zhenfeng Bian', Jie Wang ${ }^{* \neq,}$, , Zhong Lin Wang *t, ,\#

Beijing Institute of Nanoenergy and Nanosystems, Chinese Academy of Sciences, Beijing 100083, China

$\S$ School of Nanoscience and Technology, University of Chinese Academy of Sciences, Beijing 100049, China

†Education Ministry Key and International Joint Lab of Resource Chemistry and Shanghai Key Lab of Rare Earth Functional Materials, Shanghai Normal University, Shanghai 200234, China

\#School of Materials Science and Engineering, Georgia Institute of Technology, Atlanta, GA 30332, USA

\section{Corresponding Authors}

*E-mail: wangjie@binn.cas.cn

*E-mail: zhong.wang@mse.gatech.edu

\section{Author Contributions}

Linglin Zhou, Li Liu, and Wenyan Qiao contributed equally to this work. 


\section{Supporting Notes}

Note S1: The working mechanism for CS-TENG

As shown in Figure S1, due to the different electron affinities, the surface of Al and FEP film will carry the same amount and opposite charges when they contact with each other. During contacting or separating process, the potential changes between electrodes, and thus external current flow is produced with an external circuit connected.

Note S2: The removal efficiency of XO or water-oil emulsion

The removal efficiency was calculated as follow:

$$
\text { Removal efficiency }=\left(\mathrm{C}_{0}-\mathrm{C}_{\mathrm{t}}\right) / \mathrm{C}_{0} \times 100 \%
$$

where the $\mathrm{C}_{0}$ and $\mathrm{C}_{\mathrm{t}}$ are the initial and resulting concentrations of $\mathrm{XO}$ or water-oil emulsion when some charges have been consumed, respectively. Here, the removal efficiency is related to consumed charges rather than time. 


\section{Supporting Figures}

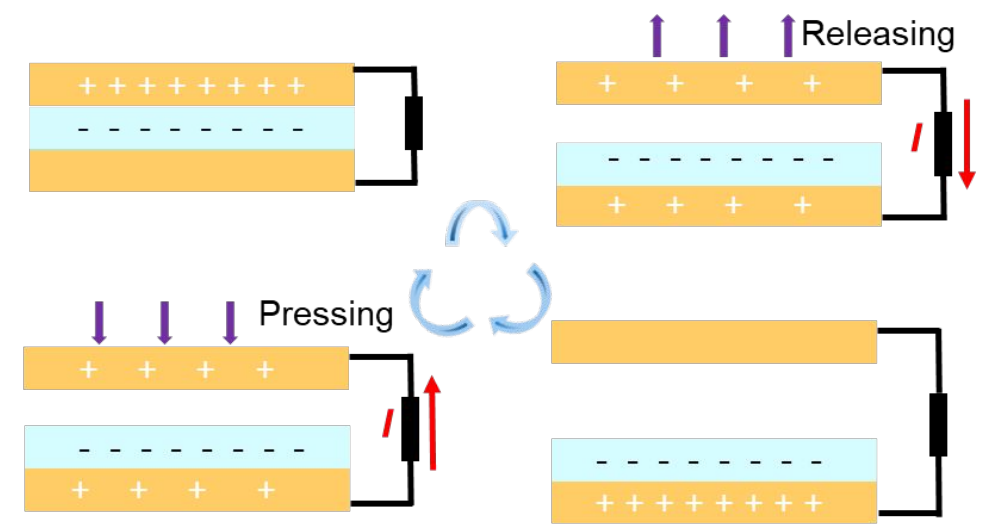
Acrylic
Foam
Al
FEP

Figure S1. The working mechanism of CS-TENG.

(a)

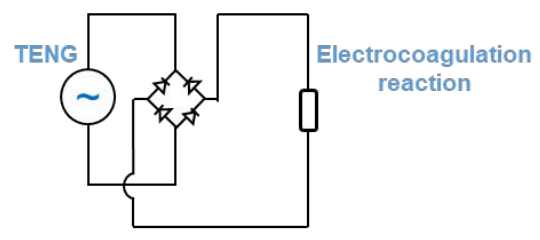

(b)

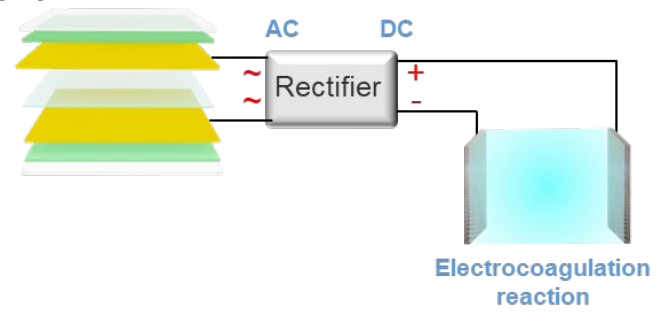

(c)

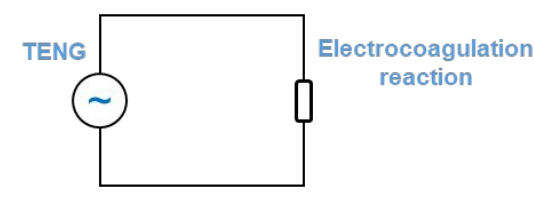

(d)

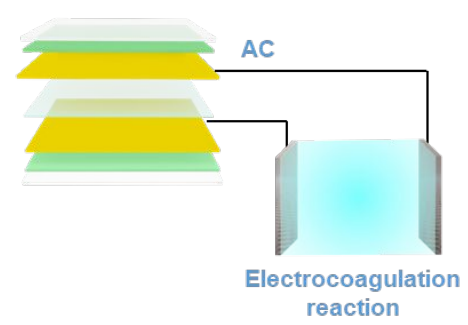

Figure S2. The circuit diagrams of self-powered electrocoagulation system. (a) Circuit diagram and (b) schematic of the self-powered DC-electrocoagulation system. (c) Circuit diagram and (d) schematic of the self-powered AC-electrocoagulation system. 


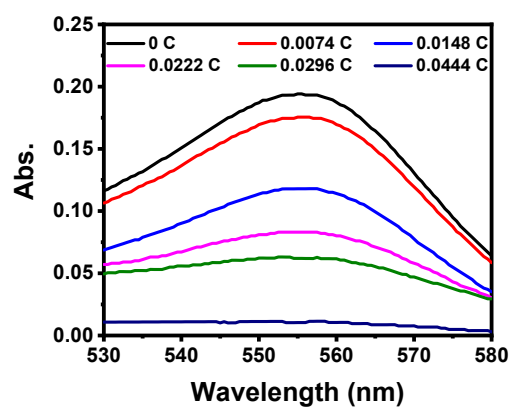

Figure S3. The UV-Vis absorption spectra of XO under DC condition at $1.8 \mathrm{~Hz}$.

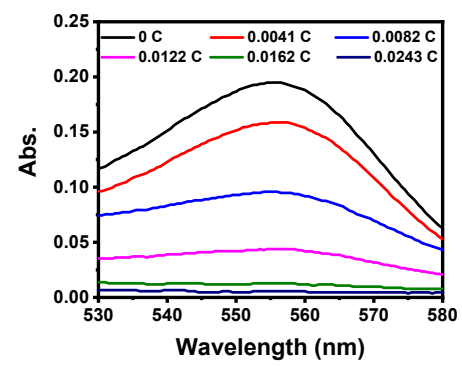

Figure S4. The UV-Visible absorption spectra of XO under AC condition at $1.8 \mathrm{~Hz}$.

(a)

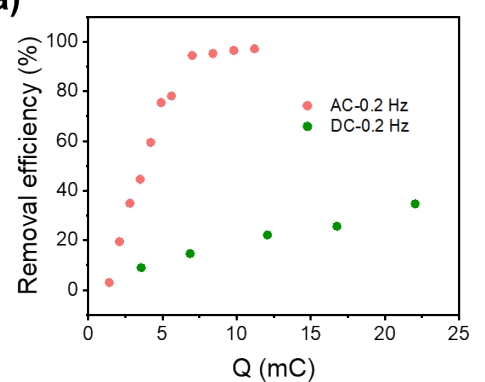

(b)

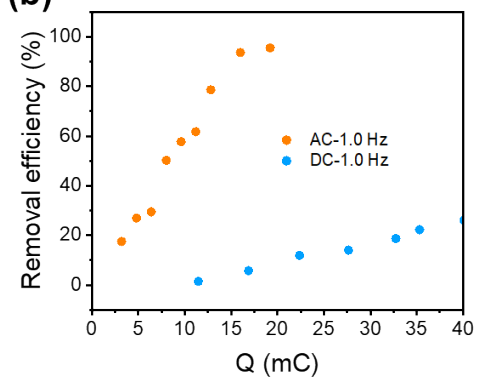

Figure S5. The removal efficiency of XO by using different power source. (a) The removal efficiency of $\mathrm{XO}$ at $0.2 \mathrm{~Hz}$. (b) The removal efficiency of $\mathrm{XO}$ at $1.0 \mathrm{~Hz}$. 
(a)

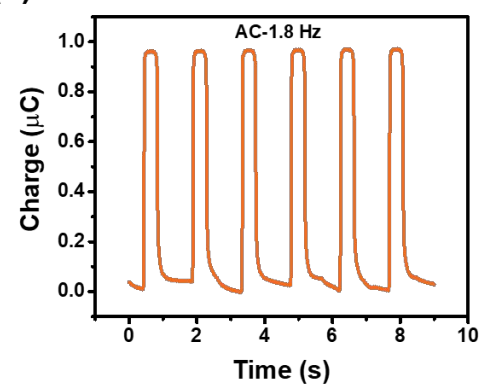

(b)

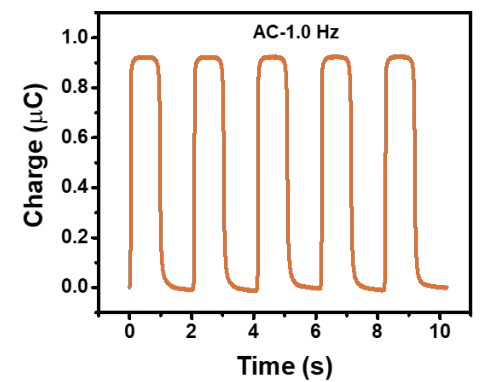

(c)

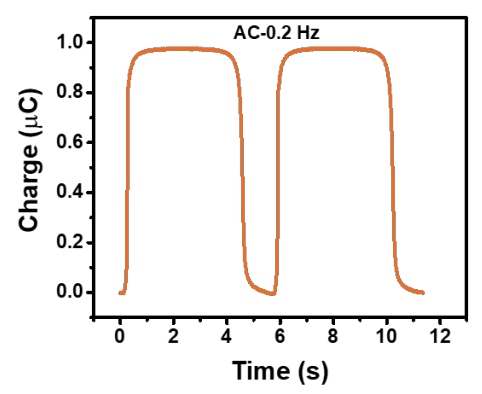

Figure S6. The transferred charge output of CS-TENG at different AC working frequencies. (a) The transferred charge output of CS-TENG at $1.8 \mathrm{~Hz}$. (b) The transferred charge output of CS-TENG at $1.0 \mathrm{~Hz}$. (c) The transferred charge output of CS-TENG at $0.2 \mathrm{~Hz}$.

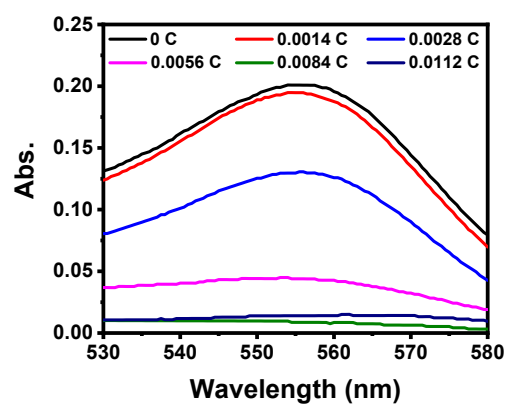

Figure S7. The UV-Visible absorption spectra of XO when consumes different charges under $\mathrm{AC}$ condition at $0.2 \mathrm{~Hz}$. 


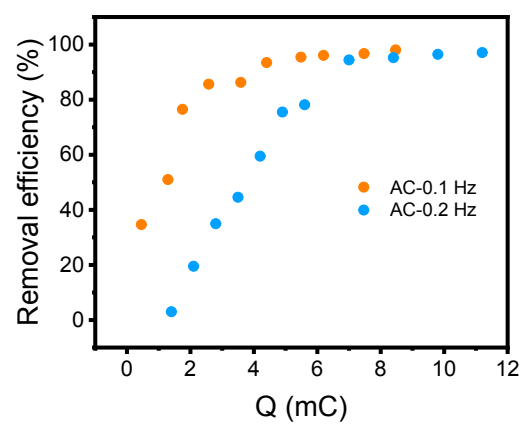

Figure S8. The removal efficiency of $\mathrm{XO}$ with the concentration of $5 \mathrm{mg} \mathrm{L}^{-1}$ under AC with $0.1 \mathrm{~Hz}$ and $0.2 \mathrm{~Hz}$.

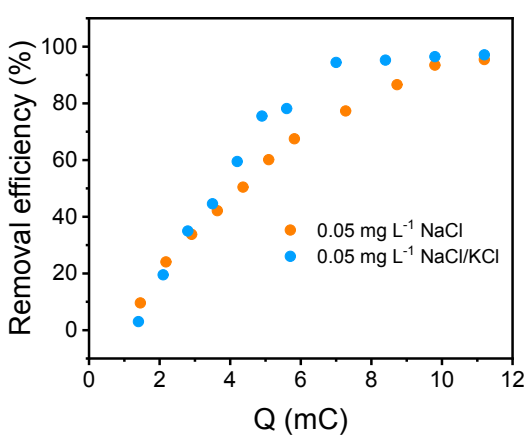

Figure S9. The removal efficiency of XO by using $0.2 \mathrm{~Hz} \mathrm{AC}$ with different electrolyte solution.

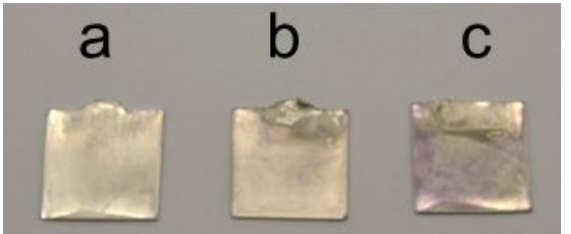

Figure S10. The photograph of $\mathrm{Al}$ electrode during the electrocoagulation reaction. (a) The photograph of original Al electrode. (b) The photograph of Al electrode after XO treatment at AC- $0.2 \mathrm{~Hz}$ of TENG. (c) The photograph of Al electrode after XO treatment at $\mathrm{AC}-1.8 \mathrm{~Hz}$ of TENG. 
(a)

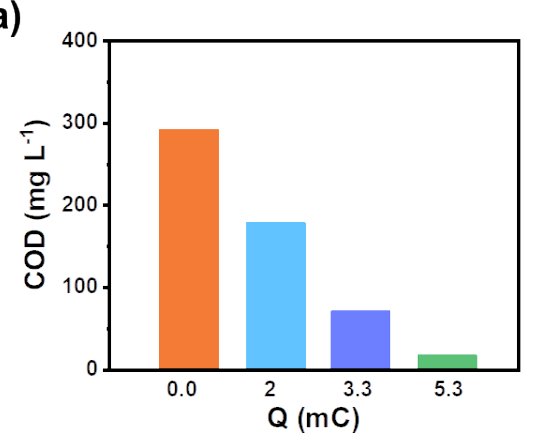

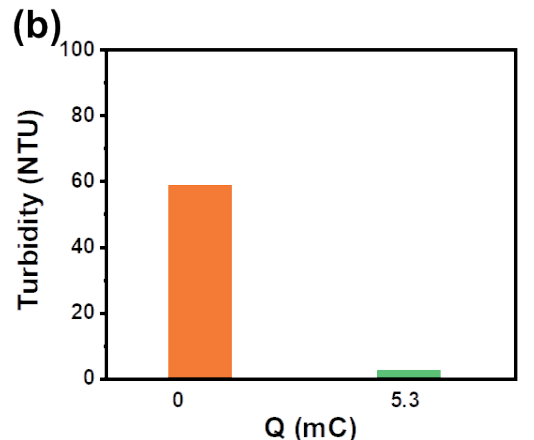

Figure S11. Electrocoagulation system for removing water-oil emulsion driven by TENG. (a) The COD value of water-oil emulsion with initial concentration of $100 \mathrm{mg}$ $\mathrm{L}^{-1}$ treating by $\mathrm{AC}$ at $0.2 \mathrm{~Hz}$. (b) The turbidity of water-oil emulsion in the initial and final states treating by $\mathrm{AC}$ at $0.2 \mathrm{~Hz}$. 